\title{
El triángulo de la educación
}

\section{The education triangle}

\author{
Karla Asúnsolo Olvera ${ }^{1}$ \\ Arlette Alvarado Ramírez ${ }^{2}$ \\ Luisa Comaduran Weckmann ${ }^{3}$
}

\begin{abstract}
Resumen
El siguiente articulo expone un proyecto de intervención socioeducativa realizado por maestras en formación de la Escuela Normal del Estado de Chihuahua Profesor Luis Urías Belderráin, llevado a cabo en un Jardín de niños al sur de la ciudad de Chihuahua, Chih. En donde se realizaron actividades dirigidas a los sujetos inmersos en el triángulo educativo: maestros, alumnos y padres de familia. Este con la finalidad de atender la problemática de la falta de involucramiento y compromiso por parte de los padres de familia así como la falta de información del personal de la escuela. Se utilizaron herramientas de carácter cuantitativo con enfoque descriptivo.
\end{abstract}

\section{Palabras clave}

Involucramiento familiar, compromiso, padres de familia.

\begin{abstract}
This presentation shows a project that took place on a preschool at the south of the city of Chihuahua, Chih. This project was made by teachers in training of the school Escuela Normal del Estado de Chihuahua Profesor Luis Urías

${ }^{1}$ Karla Asúnsolo Olvera. Institución Benemérita y Centenaria Escuela Normal del Estado de Chihuahua Profesor Luis Urías Belderráin, México. Correo electrónico: asunsolokarla@gmail.com

ID: http://orcid.org/0000-0003-3503-5492

${ }^{2}$ Arlette Alvarado Ramírez. Institución Benemérita y Centenaria Escuela Normal del Estado de Chihuahua Profesor Luis Urías Belderráin, México. Correo electrónico: arlettefernandaa@gmail.com

ID: http://orcid.org/0000-0002-2215-7442

${ }^{3}$ Luisa Comaduran Weckmann. Institución Benemérita y Centenaria Escuela Normal del Estado de Chihuahua Profesor Luis Urías Belderráin, México. Correo electrónico: l comaduranw@hotmail.com
\end{abstract}

ID: http://orcid.org/0000-0001-5480-0394 
Belderráin, in which parents, school staff and students participated. This was made with the purpose of caring about a problematic about the lack of family engagement and commitment from parents, as well as the lack of information of school staff. Quantitative tools were used and also a descriptive emphasis.

\section{Keywords}

Family involvement, commitment, parents.

\section{Introducción}

El presente proyecto tiene la finalidad de exponer una problemática implícita en un Jardín de niños de la ciudad de Chihuahua y las medidas que se llevaron a cabo para su mejora. Primeramente es imprescindible definir el término de involucramiento familiar, Según la NAFSCE 2018 (National Association For Family, School and Community Engagement Working Group):

El involucramiento familiar es una responsabilidad compartida en la que las escuelas y otras agencias y organizaciones comunitarias se comprometen en conjunto para involucrar a las familias de manera significativa y en la que las familias se comprometan a apoyar activamente el aprendizaje y el desarrollo de sus hijos. Es continuo a lo largo de la vida de un niño y conlleva un compromiso duradero, pero cambiando los roles de los padres a medida que los niños maduran hasta la adultez temprana.

Son muchos los estudios que demuestran los beneficios y la importancia del involucramiento familiar en el desarrollo del niño e incluso en el ambiente escolar. Según Christenson, Godber y Anderson (2005) algunos esos beneficios son: mejora del logro académico, mejor comportamiento, mayor cumplimiento de tareas, incremento de la participación en clases, mejora de la autoestima, mayor asistencia a clases y probabilidad de terminar la educación básica. Por lo mencionado anteriormente se consideró de suma importancia tomar medidas e intervenir para una mejora a través de un proyecto de intervención.

\section{Desarrollo}

Este apartado se dividió en ocho subtemas: primero se expone el contexto de la zona donde se encuentra la institución, en el segundo apartado, el diagnostico donde se da a conocer la forma en que detectamos la problemática; el tercero es el planteamiento del problema, el cual contiene la problemática de investigación, el objetivo general y los específicos. El cuarto es la metodología, la cual es un recopilación de estrategias que se utilizaron, la forma en que se aplicaron, instrumentos y enfoque; el quinto apartado 
muestra los resultados donde se refleja el producto del trabajo elaborado y en el sexto apartado, la conclusión en donde se plasma si se alcanzaron los objetivos o no, mas observaciones y comentarios de dichos resultados.

\section{Contexto}

El Jardín de niños está ubicado al sur de la ciudad de Chihuahua, Chih. Se considera una escuela estructuralmente pequeña, debido al tamaño de la misma, esta carece de áreas verdes, espacios amplios para que los niños jueguen, posibilidad de ampliación o construcción de más aulas, etc.

La institución cuenta con los servicios de luz, agua, teléfono e internet. El nivel socio económico de las familias que conforman la escuela varía entre medio-bajo y bajo. Los tipos de trabajo al que se dedican los padres de familia son diversos, destacan las amas de casa, trabajadores de maquila, negocios propios, algunos profesionistas y empleados. En general la comunidad cuenta con todos los servicios, las calles están pavimentadas y a los alrededores de la colonia se encuentran diversos negocios.

\section{Diagnóstico}

Primeramente se realizó una visita previa al Jardín de niños con el propósito de identificar necesidades y áreas de oportunidad para trabajarlas y llevar a cabo un proyecto de intervención como ruta de mejora. En la visita se estableció un diálogo con el colectivo del Jardín, incluida la directora, maestras titulares, trabajadora manual y personal de unidad de servicio y apoyo a la educación regular (USAER); todas ellas expresaron su inconformidad hacia la falta de compromiso y participación de los padres de familia en relación al desarrollo escolar de sus hijos.

Acto seguido se pretendió hacer un diagnóstico para determinar si realmente el involucramiento familiar era una problemática, por lo que se mandaron encuestas anónimas a casa (Anexo 1), las cuales fueron respondidas exclusivamente por los padres o tutor del niño. Después se realizó un análisis de datos, el cual se muestra en el apartado de resultados (Figura 1, 2, y 3).

\section{Problemática}

Tras analizar las características y necesidades del Jardín de niños y después de haber realizado un diagnóstico, se determinó una problemática en específico que afecta al desarrollo de los niños y al trabajo de las maestras, esta es la ausencia y falta de involucramiento de los padres de familia en las actividades de la escuela como festivales, juntas, actividades de trabajo padre-hijo, etc. 
Las maestras titulares comentaron que los padres de familia no muestran mucho interés, seriedad y compromiso hacia las actividades mencionadas anteriormente; esperan llegar a la primaria para asistir a juntas "realmente importantes". Incluso, hay padres que cuando son convocados a ir por el diagnóstico de sus hijos, prefieren no llevarlos a clases con tal de no tener que quedarse un poco de tiempo en el plantel.

Esto afecta al desarrollo cognitivo, actitudinal y muy en especial, al desarrollo emocional del niño. Si se reflexionara la niñez de cada uno y se analizara qué tanto afectó la presencia de los padres de familia, su actitud hacia los logros, sus expectativas, su reacción ante situaciones diversas, de qué manera la escuela favorecía o no la convivencia con los padres, entre otros factores, se puede establecer más empatía hacia los niños. El tiempo genera cambios en la sociedad, gracias a las tecnologías, investigaciones, etc. pero si algo se puede afirmar es que el rol de la familia y la escuela en el niño son imprescindibles y si estos trabajan juntos para beneficio de su desarrollo, habrá mejores resultados tanto cognitivos como emocionales, actitudinales y procedimentales.

Solís (2017) Cita a Henderson, Mapp, Jhonson \& Davies (2007) quienes mencionan que "En las escuelas donde se logra que las familias estén comprometidas en involucrarse, los alumnos obtienen beneficios significativos; de igual forma, el colegio se beneficia al tener mayor nivel de respeto y confianza entre la comunidad".

Por lo anterior, se llegó a un cuestionamiento clave para el desarrollo del proyecto: desde las posibilidades como maestras en formación ¿Cómo se puede contribuir para sensibilizar acerca de la importancia del involucramiento familiar?

\section{Objetivos}

El objetivo general fue fomentar la importancia de la participación de los padres de familia, para lograr el óptimo crecimiento y desarrollo de los alumnos. Así mismo se plantearon objetivos más específicos, el primero fue concientizar al personal de la escuela acerca de los beneficios que conlleva el trabajo colaborativo con los padres de familia, y el segundo, sensibilizar a los padres de familia para que se asuma un mayor compromiso e involucramiento hacia las actividades en relación al desarrollo de su hijo.

\section{Metodología}

Después de que se delimitó la problemática, se buscó el mejor paradigma para llevar a cabo los objetivos. Se eligió la animación sociocultural, el cual "es una actuación intencional para transformar las actitudes individuales y 
colectivas mediante la práctica de actividades sociales, culturales y lúdicas, hechas de un modo participativo" (Blanco, 2010).

El proyecto "Triángulo de la educación" consistió en un taller que se llevó a cabo el 16 de abril del 2018 el cual tuvo distintos momentos. Primeramente se tomaron en cuenta los datos obtenidos en el diagnóstico y en base a ellos se impartió a los padres de familia y personal del plantel, una conferencia por una especialista certificada por la Universidad de Harvard en el tema de Family Engagement (involucramiento familiar). La conferencia comenzó con preguntas reflexivas como ¿Cómo ven a su hijo en 15 años en base a como es ahora? ¿Habrá terminado el bachillerato? ¿Creen que tenga una educación superior? Después la conferencista habló sobre la importancia de involucrarse y maneras de hacerlo, así mismo les mencionó a las maestras distintas maneras de involucrar a los padres y el impacto de hacerlo.

A la par de que los padres escuchaban la conferencia, los niños hicieron una actividad en el aula sobre pilares de su vida con las maestras practicantes. Al terminar sincronizadamente ambas partes sus respectivas actividades, se llevó a cabo un taller de convivencia padre-hijo-maestra en que se realizaron actividades para estimular el vínculo entre ellos. La primera actividad fue un momento padre/madre- hijo, en el que el hijo le hizo un dibujo expresando sus sentimientos y así mismo el padre o la madre le hizo una carta, cuando terminaron ambos compartieron su trabajo. Fue muy emotivo ver que muchos padres y alumnos lloraron o se alegraron mucho, se pudo percibir un cambio positivo en el ambiente del aula.

La siguiente actividad incluyó a la maestra titular del grupo. El alumno debía decirle a su padre o madre todo lo que le quisiera expresar a la maestra y los adultos escribían a modo de carta todo lo que el niño dijera. Al finalizar las maestras titulares las leyeron y muchas de ellas se mostraron muy conmovidas y agradecidas. Para terminar con el taller se les brindó a los padres de familia una escala estimativa para valorar la utilidad del taller (Anexo 4) así mismo una encuesta a los niños (Anexo 3) adaptada a su nivel cognitivo y perceptivo, a las maestras también de les brindó una encuesta totalmente anónima en la que evaluaban su práctica en relación a la temática del proyecto (Anexo 2).

El alcance del proyecto fue descriptivo porque narró las impresiones de la familia, alumnos y maestros. Se utilizó un enfoque cuantitativo ya que las encuestas llevadas a cabo fueron de carácter cerrado y para la elaboración de las mismas se tomó en cuenta la tipología de Epstein. (EPSTEIN, 2011) Identifica seis formas en que las familias participan en la educación de los hijos: 
(I)crianza, facilitan en los hijos la adquisición de hábitos y valores relacionados con el logro escolar; (II) comunicación con la escuela, comparten información con docentes y directivos acerca del hijo y el funcionamiento de la escuela; (III) supervisión del aprendizaje, apoyan desde el hogar el aprendizaje del currículo escolar; (IV) toma de decisiones, participan en instancias involucradas en decisiones relativas a la escuela; (V) voluntariado, se involucran actividades que favorecen la gestión escolar y/o el aprendizaje de los estudiantes y (VI) colaboración con la comunidad.

\section{Resultados}

La encuesta realizada para el diagnóstico contenía preguntas que identificaban la percepción de los padres acerca de su involucramiento familiar como por ejemplo ¿Asiste a las juntas de su hijo(a)? ¿Se acerca a la docente para preguntar sobre el comportamiento y aprendizaje de su hijo(a)? ¿Ayuda a su hijo(a) en las tareas? Entre otras preguntas. Todas se contestaban con base a la escala de Likert, las posibles respuestas eran: siempre, frecuentemente, algunas veces, pocas veces y nunca.

Los resultados de la encuesta diagnóstico para padres de familia fueron clasificados por grados. En primer año se obtuvo un $65.8 \%$ en el equivalente a la respuesta de siempre, un $25.11 \%$ en el inciso de frecuentemente, $10.6 \%$ algunas veces, $4.2 \%$ pocas veces y solo un $3.6 \%$ en la respuesta de nunca (Figura 1). En segundo año se obtuvo que un $58.4 \%$ de los padres de familia respondieron al inciso de siempre, $22.95 \%$ frecuentemente, $9.5 \%$ respondió algunas veces, $7.5 \%$ pocas veces y un $1.5 \%$ en la respuesta de nunca (Figura 2). Las respuestas de tercer grado fueron un $55.3 \%$ siempre, $26.2 \%$ frecuentemente, $10.6 \%$ algunas veces, un $3.6 \%$ algunas veces y $4.2 \%$ nunca (Figura 3).

Figura 1.

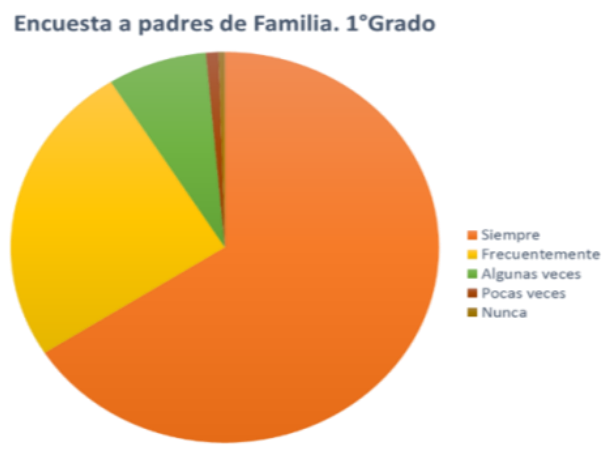

Asúnsolo Olvera, K.; Alvarado Ramírez, A.; y Comaduran Weckmann, L. 
Figura 2.

Encuesta a padres de Familia. $2^{\circ} \mathrm{Grado}$

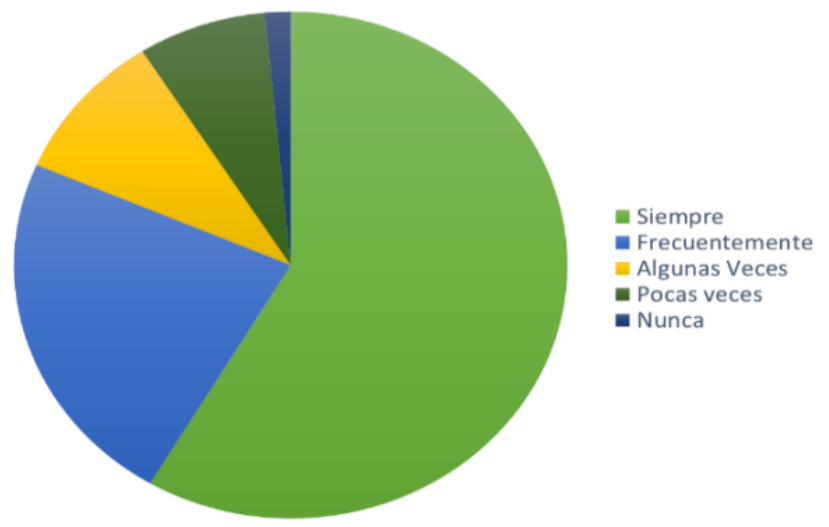

Figura 3.

Encuesta a padres de Familia. $3^{\circ} \mathrm{Grado}$

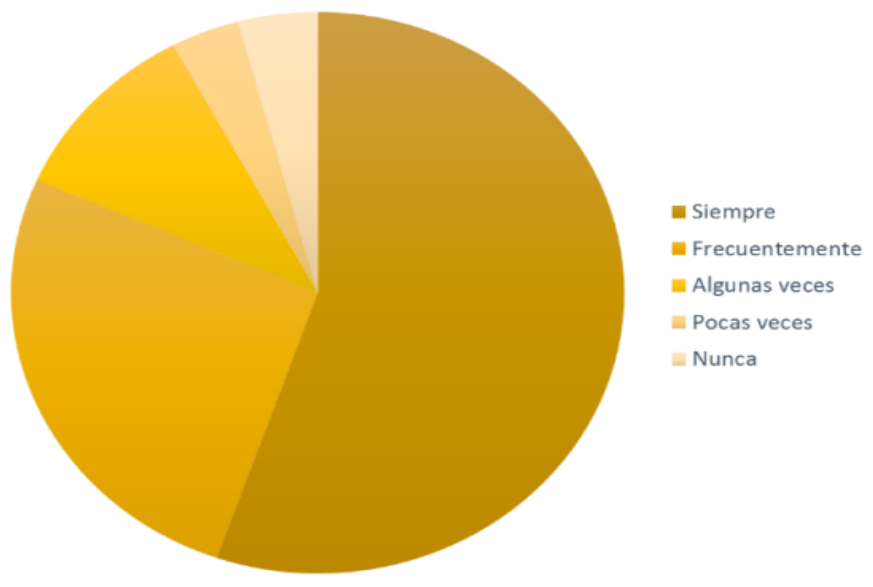

La escala estimativa de los padres de familia para valorar el taller arrojó que más del noventa y cinco por ciento consideró que la información brindada en el taller era muy relevante. Así mismo todos respondieron que era muy importante asistir a los eventos de la escuela, tener una relación constante con la maestra de su hijo y motivar a sus hijos en casa.

A diferencia de las otras preguntas con respuestas muy favorables hacia el taller, en la pregunta ¿Qué tanto se involucra como voluntario en los eventos escolares? La mayoría de las respuestas estuvo entre algo, poco y 
nada. Solo un treinta y siete por ciento contestó que siempre son voluntarios. También, hubo algunos padres que consideran que no es tan importante el involucramiento de los padres para la toma de decisiones relativas a la escuela, aunque esta fue una minoría.

Finalmente, de encuesta que se les aplicó a los alumnos seleccionamos ciertas preguntas para mejor visualización de resultados. (Figura 4).

Figura 4.

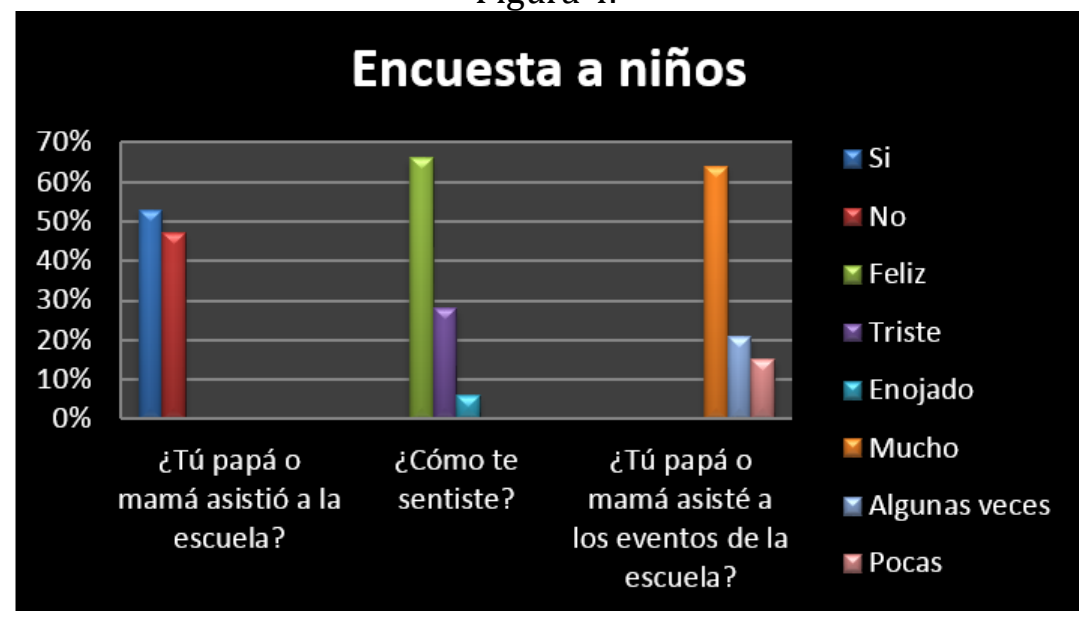

A pesar de que un $53 \%$ del alumnado estuvo acompañado por sus padres durante el taller y un $47 \%$ del alumnado no estuvo acompañado por sus padres, un $66 \%$ se sintió feliz por haber estado acompañado o no por sus padres, un $28 \%$ se sintió triste por no haber estado acompañado por sus padres y un $6 \%$ se sintió enojado. Cabe mencionar que la encuesta se le aplicó a todo el alumnado, incluidos aquellos niños que no participaron por la ausencia de sus padres.

Así mismo se les preguntó quién hacía con ellos la tarea, quién les enseña que deben portarse bien, entre otras. Los resultados muestran que la respuesta varió entre padres y abuelos, en muy pocos casos, hermanos.

Por último se entregó una encuesta a las maestras titulares de opción múltiple (Anexo 2) donde tenían que responder en qué nivel promueven en práctica la participación de los padres de familia. De dicha encuesta se obtuvo como resultado $66 \%$ en la respuesta equivalente a algunas veces y un $33 \%$ en la respuesta de casi siempre. Así mismo en la pregunta referente a si permiten que los padres de familia participen en la toma de decisiones, la respuesta en su mayoría fue a veces y casi nunca, lo que indica que falta 
conciencia y motivación por parte de las maestras titulares en cuestión de promover acciones que beneficien a dicha causa.

\section{Conclusión}

En conclusión y haciendo referencia a las encuestas, podemos afirmar que los resultados fueron más favorables para el grupo de primero, es decir los padres de familia tuvieron una respuesta muy positiva en la asistencia del taller y por ende en las actividades llevadas a cabo. Así mismo, los padres de familia de tercer grado fueron los que menos asistieron al taller, y esto se pudo ver reflejado en las encuestas de los niños, que respondieron sentirse tristes con respecto a la inasistencia de sus padres. También, creemos que las encuestas utilizadas para el diagnóstico no fueron contestadas con sinceridad a pesar de que eran anónimas, ya que los resultados no coincidían con la realidad.

El taller lo consideramos favorable para el cumplimiento de los objetivos ya que durante la plática hubo gran interés por parte de los padres, preguntaron dónde podían buscar más información e incluso pidieron que se volviera a impartir por la tarde para que pudieran asistir sus parejas. Al terminar hubo muchos buenos comentarios y felicitaciones acerca del taller. A pesar de esto, creemos que se le debe dar un seguimiento, ya que sí se logró fomentar la importancia del involucramiento familiar pero no es algo que se logre en un solo día, es un proceso que se debe trabajar día con día hasta obtener resultados permanentes.

En general el proyecto fue una experiencia de aprendizaje y convivencia para todos que se debería de impartir en todas aquellas escuelas que quieran perseguir nuestros mismos o similares objetivos con el fin de mejorar el funcionamiento del plantel, pero más que nada, que quieran generar un ambiente de aprendizaje y desarrollo para los niños, un ambiente donde los padres se puedan involucrar y donde las maestras y directivos trabajen en colaboración para crear un ambiente de seguridad, confianza, alegría y enseñanza para todos.

\section{Referencias}

Blanco, M. C. (2010). Concepto, objetivos y funciones de la animación sociocultural. Innovación y Experiencias Educativas.

Christenson, S. G. (2005). School-family partnerships for childrens succes. Nueva York: Teachers College Press.

EPSTEIN, J. (2011). School, family, and community partnerships. New York: Westview Press. 
NAFSCE. (05 de mayo del 2018). NAFSCE. Obtenido de https://www.nafsce.org/page/definition

Solís Castillo Fernando, A. S. (2017). Análisis del papel del involucramiento de la familia en la escuela secundaria y su repercusión en el rendimiento académico.

\section{Anexos}

Anexo 1. Encuesta de diagnóstico a padres de familia GRADO QUE CURSA SU HIJ@: ¿Asiste a las juntas de su hijo(a)?
a) Siempre
b) Frecuentemente ¿Por qué?
c) Algunas veces
d) Pocas veces e) Nunca

Asiste a las actividades del jardín de niños, por ejemplo: festejos del día del niño, kermesse, festivales de la primavera, revolución, etc.
a) Siempre
b) frecuentemente
c) algunas veces
d) pocas veces
e) nunca

A la hora de salida ¿quién recoge usualmente a los niños?
a) Padres de familia
b) abuelos
c) guardería
d) vecinos u otros
e) niñera

¿Con qué frecuencia recoge usted a su hijo(a) de la escuela?
a)Siempre
b) frecuentemente
c) algunas veces
d) pocas veces
e) nunca ¿Por qué? (en caso de ser por trabajo, especifique su horario laboral)

¿Se acerca a la docente para preguntar sobre el comportamiento y aprendizaje de su hijo(a)?
a) Siempre
b) frecuentemente
c) algunas veces
d) pocas veces
e) nunca

¿Ayuda a su hijo(a) en las tareas?
a) Siempre
b) frecuentemente
c) algunas veces
d) pocas veces
e) nunca

¿Platica con la docente sobre alguna inquietud que manifiesta su hijo(a)?
a) Siempre
b) frecuentemente
c) algunas veces
d) pocas veces
e) nunca

¿Mantiene comunicación con la maestra de su hijo(a)?
a) Siempre
b) frecuentemente
c) algunas veces
d) pocas veces
e) nunca 
¿Participa en las actividades organizadas para el mejoramiento de la escuela?
a) Siempre
b) frecuentemente
c) algunas veces
d) pocas veces
e) nunca

¿Platica con su hijo(a) acerca de sus compañeros de la escuela?
a) Siempre
b) frecuentemente
c) algunas veces
d) pocas veces
e) nunca

¿Platica con su hijo(a) acerca de lo que hizo en la escuela?
a) Siempre
b) frecuentemente
c) algunas veces
d) pocas veces
e) nunca

Considera que es convocado por la maestra:
a) Con mucha frecuencia
b) lo necesario
c) pocas veces
e) nunca

\section{Anexo 2. Encuesta a docentes}

1. Desarrolla proyectos de padres voluntarios para apoyar a la escuela y a otros padres
0 . Nunca
1) Casi nunca
2) A veces
3) Casi siempre
4) Siempre

2. Informa acerca de programas (salud, culturales) de la comunidad que apoya a los estudiantes
0 . Nunca
1) Casi nunca
2) A veces
3) Casi siempre
4) Siempre

3. Promueve que los padres participen en actividades en la comunidad que apoyan el aprendizaje de los estudiantes
0 . Nunca
1) Casi nunca
2) A veces
3) Casi siempre
4) Siempre

4. Permite que los padres participen en las decisiones escolares
0 . Nunca
1) Casi nunca
2) A veces
3) Casi siempre
4) Siempre

5. Informa de los métodos para evaluar el desempeño de los estudiantes
0 . Nunca
1) Casi nunca
2) A veces
3) Casi siempre
4) Siempre

6. Entrega reportes de logros y dificultad académica informa las políticas y normas escolares.
0 . Nunca
1) Casi nunca
2) A veces
3) Casi siempre
4) Siempre

7. Informa las políticas y normas escolares
0 . Nunca
1) Casi nunca
2) A veces
3) Casi siempre
4) Siempre 
8. Orienta como mejorar la motivación por el aprendizaje
0 . Nunca
1) Casi nunca
2) A veces
3) Casi siempre
4) Siempre

9. Orienta como apoyar a los hijos con sus tareas en casa
0 . Nunca
1) Casi nunca
2) A veces
3) Casi siempre
4) Siempre

10. Enseña estrategias para apoyar el estudio de su hijo
0 . Nunca
1) Casi nunca
2) A veces
3) Casi siempre
4) Siempre

Anexo 3. Encuesta a alumnos

¿Tu papá vino a la escuela hoy?
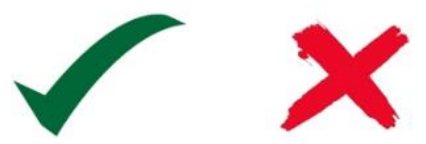

¿Cómo te sentiste?
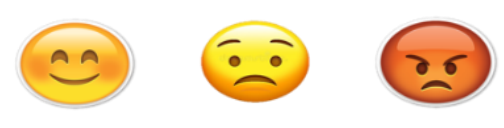

¿Quién te ayuda a hacer tu tarea?
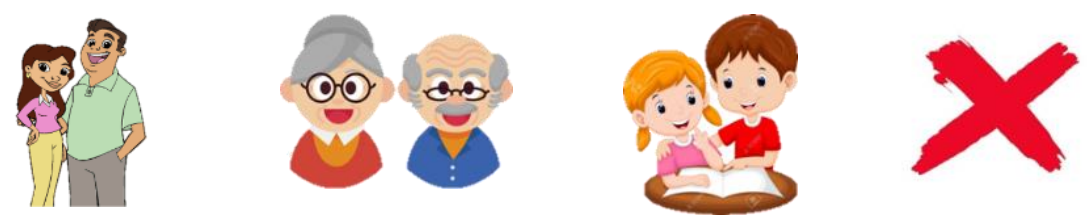

¿Tu mamá o papá asiste a tus eventos de la escuela?
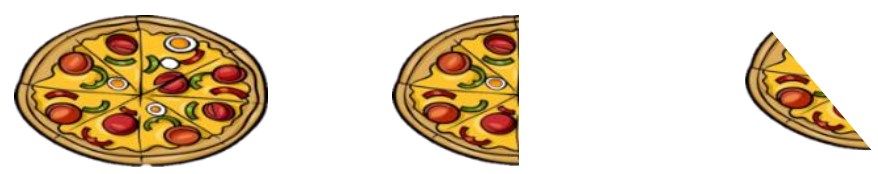

¿Tu mamá o papá juega en casa o va al parque contigo?
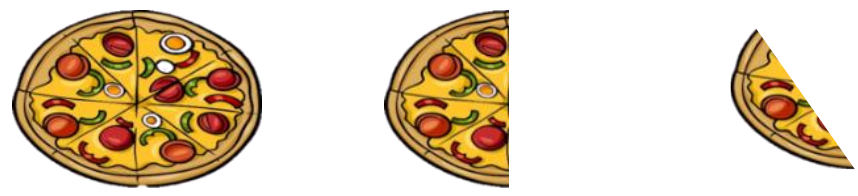

Asúnsolo Olvera, K.; Alvarado Ramírez, A.; y Comaduran Weckmann, L. 
¿Platicas con mamá o papá acerca de lo que hiciste en la escuela?
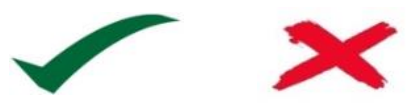

¿Quién te enseña cómo debes de portarte?
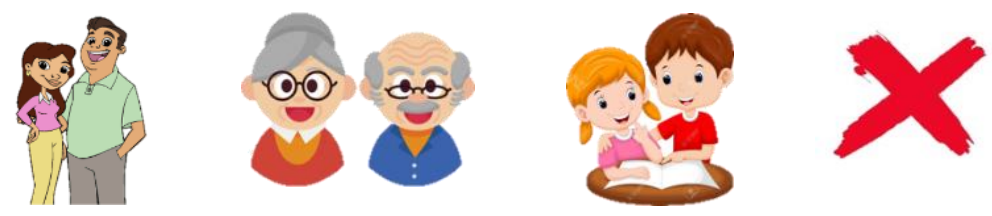

Tus papás te quieren:
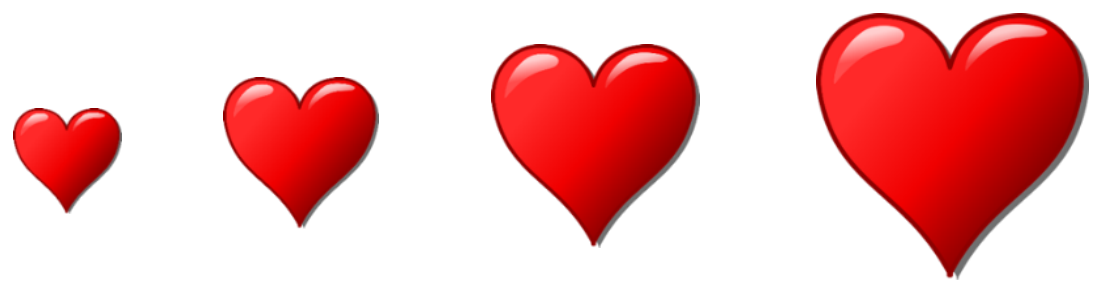

Anexo 4. Encuesta a padres de familia

1. ¿Qué tan relevante cree que es la información brindada en el taller? Mucho Algo Poco Nada

2. ¿Cree que es importante para el desarrollo de su hijo asistir a los eventos de la escuela?
Mucho
Algo
Poco
Nada

3. ¿Qué tan importante considera que es motivar a su hijo en asuntos relativos a la escuela?
Mucho
Algo
Poco
Nada

4. ¿Qué tan importante considera la comunicación maestro-padre de familia?
Mucho
Algo
Poco
Nada 
5. ¿Qué tan importante considera que es el involucramiento de los padres en la toma de decisiones relativas a la escuela?
Mucho
Algo
Poco
Nada

6. ¿Qué tanto se involucra como voluntario en los eventos escolares?

Mucho Algo Poco Nada

7. ¿Qué tan dispuesto esta en comprometerse con su hijo después de la información brindada en el taller?
Mucho
Algo
Poco
Nada

8. ¿Qué tan importante cree que es para su hijo la motivación desde casa?
Mucho
Algo
Poco
Nada

¡Gracias! 